\title{
Expert Consensus on Screening and Assessment of Cognition in Psychiatry
}

\author{
Roger S. McIntyre (iD, ${ }^{1,2 * N i c o l e ~ A n d e r s o n, ~}{ }^{3}$ Bernhard T. Baune, ${ }^{4}$ Elisa Brietzke (iD, ' \\ Katherine Burdick, ${ }^{5}$ Phillipe Fossati, ${ }^{6}$ Philip Gorwood iD, ${ }^{7}$ Catherine Harmer, ${ }^{8}$ John \\ Harrison, ${ }^{9}$ Philip Harvey, ${ }^{10}$ Rodrigo B. Mansur, ${ }^{2}$ Alice Medalia, ${ }^{11}$ Kamilla \\ Miskowiak, ${ }^{12}$ Tanya Ramey, ${ }^{13}$ Carola Rong, ${ }^{2}$ Joshua D. Rosenblat, ${ }^{2}$ Allan Young, ${ }^{14}$ and \\ Stephen M. Stahl ${ }^{15}$
}

\footnotetext{
${ }^{1}$ Department of Psychiatry and Pharmacology, University of Toronto, Toronto, Ontario, Canada

${ }^{2}$ Mood Disorders Psychopharmacology Unit, University Health Network, Toronto, Ontario, Canada

${ }^{3}$ Rotman Research Institute at Baycrest, Toronto, Ontario, Canada

${ }^{4}$ Department of Psychiatry, The University of Melbourne, Melbourne, Australia

${ }^{5}$ Department of Psychiatry, Brigham and Women's Hospital, Boston, MA, USA

${ }^{6}$ Department of Psychiatry, Ecole des Neurosciences Paris Ile-de-France, Paris, France

${ }^{7}$ Centre de Psychiatre et de Neurosciences, Hopital Saint-Anne (Paris Descartes University), Paris, France

${ }^{8}$ Psychopharmacology and Emotional Research Lab, University of Oxford, Oxford, England

${ }^{9}$ Metis Cognition Ltd., Warminster, UK

${ }^{10}$ Department of Psychiatry and Behavioral Sciences, University of Miami Miller School of Medicine, Miami, Florida, USA

${ }^{11}$ Department of Psychiatry, Columbia University Irving Medical Center, New York, New York, USA

${ }^{12}$ Neurocognition and Emotion in Affective Disorders, Psychiatric Center Copenhagen, Copenhagen University Hospital, Rigshospitalet

${ }^{13}$ Division of Therapeutics and Medical Consequences, National Institute on Drug Abuse, National Institutes of Health, North Bethesda, Maryland, USA

${ }^{14}$ Department of Psychological Medicine, King's College London and South London and Maudsley NHS Foundation Trust, London, UK

${ }^{15}$ Department of Psychiatry, University of California, San Diego, California, USA
}

During the past two decades, it has been amply documented that neuropsychiatric disorders (NPDs) disproportionately account for burden of illness attributable to chronic non-communicable medical disorders globally. It is also likely that human capital costs attributable to NPDs will disproportionately increase as a consequence of population aging and beneficial risk factor modification of other common and chronic medical disorders (e.g., cardiovascular disease). Notwithstanding the availability of multiple modalities of antidepressant treatment, relatively few studies in psychiatry have primarily sought to determine whether improving cognitive function in MDD improves patient reported outcomes (PROs) and/or is cost effective. The mediational relevance of cognition in MDD potentially extrapolates to all NPDs, indicating that screening for, measuring, preventing, and treating cognitive deficits in psychiatry is not only a primary therapeutic target, but also should be conceptualized as a transdiagnostic domain to be considered regardless of patient age and/or differential diagnosis.

Received 22 March 2018; Accepted 15 June 2018; First published online 15 January 2019

\section{Introduction}

Emerging from the literature is a consistent observation that cognitive dysfunction, a transdiagnostic psychopathological domain in psychiatry, is a principal determinant of a person's general function and other patient-reported outcomes (PROs). ${ }^{1,2}$ During the past

* Address correspondence to: Roger S. McIntyre, University Health Network - Brain and Cognition Discovery Foundation, 399 Bathurst Street, Toronto, ON, Canada M5T 2 S8.

(Email: Roger.McIntyre@uhn.ca) two decades, it has been amply documented that neuropsychiatric disorders (NPDs) disproportionately account for burden of illness attributable to chronic noncommunicable medical disorders globally. ${ }^{3}$ It is also likely that human capital costs attributable to NPDs will disproportionately increase as a consequence of population aging and beneficial risk factor modification of other common and chronic medical disorders (e.g., cardiovascular disease). ${ }^{4}$ The early age at onset, the relatively high incidence and prevalence, and the chronic and unfavorable illness trajectory, as well as the absence 
of scalable, preventable, disease-modifying and/or curative therapies for NPDs account for the staggering human, societal, and economic costs. ${ }^{5,6}$

The ignominious psychosocial and human capital consequences of NPDs have provided the impetus to identify the dimensions/domains of psychopathology that primarily mediate health outcomes among affected individuals. Whether NPDs are typologized according to severity (i.e., common, severe), age of onset of initial observable characteristics (i.e., childhood, adulthood, late life), and/or conceptual pathophysiological processes (i.e., neurodevelopmental, neurodegenerative), deficits in cognitive function account for more variance in PROs and costs attributable to NPDs than any other psychopathological domain. ${ }^{1,2,7}$

For example, major depressive disorder (MDD) debases human capital more than any other brain-based disorder among younger populations (i.e., 18-45 years). ${ }^{8}$ Epidemiological and clinical studies provide results that are in accordance with the assertion that cognitive deficits (self-rated, objectively measured) account for more variability in interpersonal adjustments and/or workplace performance (i.e., absenteeism, presenteeism) than total depression symptom severity and/or other domains (i.e., factors) in persons with mood disorders. ${ }^{9-12}$ It is additionally noted that cognitive deficits in MDD may predate the onset of "mood symptoms" in MDD and may progress in overall magnitude of deficits as a function of episode frequency/illness duration. ${ }^{13,14}$

Notwithstanding the availability of multiple modalities of antidepressant treatment, relatively few studies in psychiatry have primarily sought to determine whether improving cognitive function in MDD improves PROs and/ or is cost effective. ${ }^{10,15}$ The mediational relevance of cognition in MDD potentially extrapolates to all NPDs, indicating that screening for, measuring, preventing, and treating cognitive deficits in psychiatry is not only a primary therapeutic target, but also should be conceptualized as a transdiagnostic domain to be considered regardless of patient age and/or differential diagnosis. ${ }^{16,17}$

\section{Transdiagnostic domains}

In keeping with the view that disturbances in cognitive functions are a transdiagnostic phenomenon, the National Institutes of Health has proffered the Research Domain Criteria (RDoC), which broadly aims to provide a biobehavioral mechanistic matrix of NPDs. ${ }^{18}$ Among NPDs, deficits in general cognitive function, social cognition (i.e., theory of mind), negative cognitive valence systems (e.g., perceived threat), and positive cognitive valence systems (e.g., motivation and reward) are distributed across four of the current five RDoC domains. ${ }^{19-21}$ The RDoC framework is supported by animal and human cognitive neuroscience data indicating that domain-based psychopathology (i.e., self-report, observable characteristics) is subserved by discrete multilevel and multimodal substrates. It is expected that future discovery and development of psychiatric treatments are more likely to adopt a "domain-based" rather than a "disease-based" (e.g., bipolar disorder, schizophrenia) approach. ${ }^{22-24}$ For example, a treatment capable of ameliorating abnormalities in reward or cognition domain would not only have transdiagnostic application, but also would mimic the strategic framework of developing treatments in other chronic diseases (i.e., disease-agnostically targeting dimensions/domains).

\section{Importance of cognition as a transdiagnostic domain}

The relevance of cognitive domain disturbances as principal mediators of health outcomes across NPDs is expected to only amplify as the global economy and workforce adapt to the "human capital" or "digital" economy. ${ }^{25,26}$ Moreover, quality of care initiatives across multiple jurisdictions, as well as greater emphasis on cost effectiveness and containment, provide the impetus for the health-care ecosystem, and its stakeholders, to place greater emphasis on prevention, early intervention, risk factor modification, and specific targeting of critical determinants of health outcomes. ${ }^{27}$ Tacit to this reprioritization is the requirement for health-care providers to be familiar with a systematic approach to assessing cognitive functions as key determinants of proximal as well as distal health outcomes, agnostic of age and any preliminary differential diagnostic considerations. Hitherto, health-care providers have received extensive education in the approach to screening for (e.g., Mini Mental Status Exam [MMSE]) and diagnosing cognitive deficits in elderly populations. ${ }^{28}$ Notwithstanding this, there has been relatively less attention (and consequently, deficiencies in best practices) given to a systematic screening/assessment of cognitive function deficits (i.e., subjective and/or objective) in pediatric and/or nongeriatric populations presenting to healthcare providers, wherein cognitive disturbances are the primary focus of clinical attention.

Against this background, we aim herein to provide health-care providers with a meta-guideline for cognitive dysfunction (i.e., subjective, objective) in clinical practice with an emphasis on screening and differential diagnosis. We purposefully avoid an approach to cognitive dysfunction that is disease specific and/or gives priority to age. Instead, we approach cognitive function as informed by cognitive neuroscience: cognition is a transdiagnostic domain that should be assessed systematically regardless of differential diagnoses. ${ }^{29}$ It is recognized that cognitive dysfunctions vary across NPDs and individuals as well as within individuals as a function 


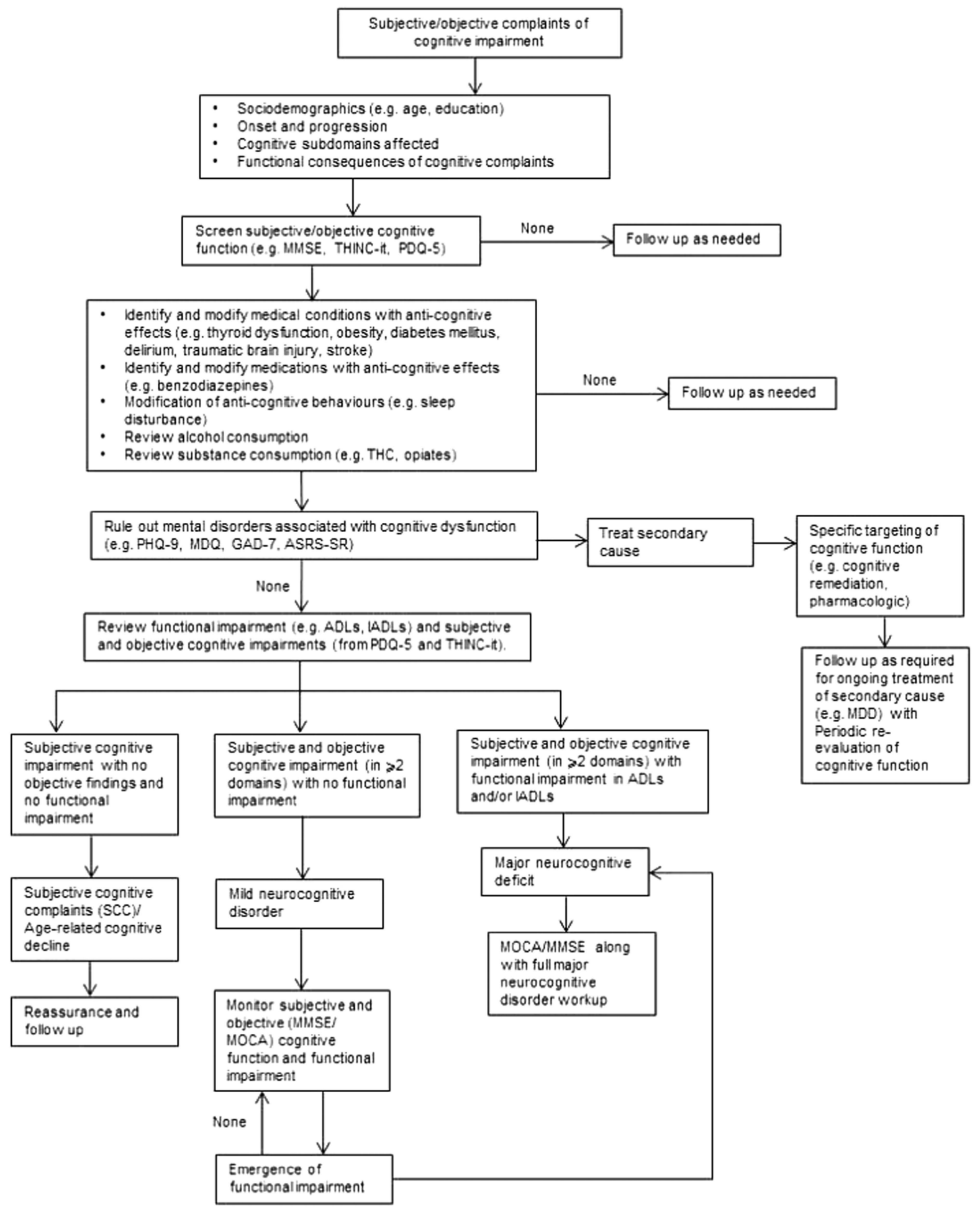

FIGURE 1. Algorithm for cognitive screening and assessment in the clinical setting.

of illness progression. It is also well established that multiple sociodemographic, clinical, and treatment factors moderate overall cognitive function as well as domain-specific performance.

It is our view that approaching psychopathology with a domain/dimensional-based approach (i.e., rather than a disease-specific approach) reflects clinical practice and is not fundamentally different than the approach taken to other targets in chronic disease regardless of etiology or diagnosis (e.g., hypertension, peripheral blood glucose). ${ }^{30,31}$ It is anticipated that a systematic approach to screening and measuring cognitive dysfunction across 
NPDs will streamline assessment, diagnosis, and care pathways, and it is hoped that this tactic will presage greater precision, consistency, appropriateness, and cost effectiveness of care.

\section{Systematic Approach to Assessing Cognition}

(Figure 1) For patients presenting with cognitive complaints as a focus of clinical concern or for whom cognitive deficits are suspected based on change in patient function, the initial step begins with ascertaining whether the cognitive deficits are subjective and/or objective. Across NPDs, it is well established that subjective and objective cognitive complaints exhibit minimal correlation. ${ }^{32-37}$ A separate body of literature indicates that changes in subjective and objective cognitive measures over time may correlate to a greater extent than pretreatment cross-sectional cognitive measures. ${ }^{38,39}$

The dissociation between subjective and objective cognitive performance is a consequence of multiple moderating factors (e.g., depressive symptoms). ${ }^{40}$ Implicit is the need for health-care practitioners to provide reassurance when cognitive deficits are solely subjective and are not verified objectively and/or associated with meaningful functional impairment. Notwithstanding this, vigilance for the possibility of objective cognitive impairment should remain in circumstances in which a patient or caregiver reports functional deficits in the absence of subjective cognitive complaints.

Available evidence also indicates that informant (e.g., family) ratings of an identified patient's cognitive function are reliable, valid, and highly correlated with both subjective and objective ratings of cognition. For example, the Cognitive Assessment Interview was derived from the Schizophrenia Cognition Rating Scale and the Clinical Global Impression of Cognition in Schizophrenia with neurocognitive battery measures, functional assessment, and functional outcome. ${ }^{41}$

Sociodemographic information with a particular emphasis on age and education are essential, and these are two of the most replicated variables influencing general cognitive performance. Early childhood adversity (ECA) has been associated with a myriad of medical and/ or mental disorders. Preclinical evidence, as well as emerging evidence in human studies, indicates that ECA may be associated with abnormalities in general cognitive functions and social cognition. It is well established that ECA exerts deleterious effects on cognitive emotional processing that overlap with positive and negative valence disturbances. ${ }^{42}$

As with all patient-reported chief complaints and/or objectively established deficits in psychosocial and/or workplace function, characterization of the onset, trajectory, progression, and course is warranted.
Determining whether the cognitive complaint/deficit is enduring or progressive has diagnostic implications (e.g., intellectual disability, bipolar disorder/major neurocognitive disorder, respectively). Moreover, cognitive complaints/objective deficits that temporally precede the overt onset of an NPD may represent heterotypic continuity, vulnerability markers, early prodrome of the NPD, or a distinct and separate disease process. Conversely, cognitive deficits may become a focus of clinical concern following the onset and/or amelioration of a discrete medical/mental disorder (e.g., traumatic brain injury, major depressive disorder) or spontaneous or medication-induced remission. ${ }^{43}$

\section{Subdomains of cognition}

Cognitive functions have been variably defined and operationalized. Delineating whether deficits in cognition are primarily in the domains of learning and memory, attention/concentration, executive functions (e.g., planning, sequencing, organizing, impulse control), processing speed, language, and/or social cognition is critical. ${ }^{1}$ It is recognized that cognitive subdomains exhibit multilinearity, yet as discrete subdomains often are subserved by discrete yet interrelated biological substrates. A separate yet overlapping taxonomy is "hot" and "cold" cognition, referring to the presence and absence of emotional valence, respectively. ${ }^{44}$ Examples of "hot" cognition include, but are not limited to, rumination, negative attentional biases, affect perception and recognition, and catastrophic reactions to real and/or perceived slights. ${ }^{45}$

Deficits in any subdomain are not pathognomonic of any single NPD or medical disorder. The profile of cognitive deficits may provide insights into possible disease considerations. For example, childhood onset of persisting attentional disturbances with difficulties in impulse control are characteristic features of attentiondeficit hyperactivity disorder, while deficits in memory (e.g., spatial) may suggest early prodromal stages of a major neurocognitive disorder when significant deficits in multiple domains of psychosocial functioning are present. ${ }^{46}$

\section{Functional consequences of alterations in cognitive performance}

The functional consequences of cognitive dysfunction are essential when attempting to establish clinical meaningfulness. To the extent possible, characterizing "premorbid" function is instructive, as many individuals with significant "cognitive reserve" may manifest significant subjective cognitive decline that may not be verifiable using norms whereby many cognitive screening and measuring tools have been validated. General 
psychosocial functioning, independence in everyday living, academic performance (where applicable), and workplace productivity/presenteeism should be evaluated. Among individuals who are working, presenteeism is essential information, as workplace productivity decreases are disproportionately accounted for by presenteeism than absenteeism. ${ }^{4}$ A review of activities of daily living (ADLs) and instrumental ADLs (IADLs) should also be conducted. A host of medical disorders are highly associated with decreased cognitive performance in both younger and older populations.

\section{Medical illness and cognition}

It is well established that thyroid dysfunction is linked to cognitive impairment and should be corrected biochemically. ${ }^{47}$ It has also emerged that impaired glucose tolerance, diabetes mellitus, and obesity are associated with impaired cognitive performance and mild cognitive impairment, as well as major cognitive disorders. ${ }^{48}$ Many other medical disorders with associated anticognitive effects should also be considered, including, but not limited to, delirium, cerebrovascular accidents, and traumatic brain injury. Finally, maladaptive behaviors impacting cognition, notably disruption in sleep duration, efficiency, and/or reversal of day/night schedule should be ruled out as modifiable contributors of cognitive impairment. ${ }^{49}$

\section{Medication effects and cognition}

Health-care providers should also review prescription and over-the-counter (OTC) medications, as well as complementary alternative medicines (CAMs), that may be associated with anticognitive effects. For example, mechanistically dissimilar agents like benzodiazepines, antihistamines, anticonvulsants, and corticosteroids are all associated with anticognitive effects. ${ }^{50,51}$ The role of anticholinergics and, of more recent interest, histamine antagonism, needs to be carefully assessed. Many OTCs with sedating/somnolent effects may have anticognitive effects, and despite marketing claims, procognitive effects associated with most CAMs have not been established. The anticognitive effects of alcohol consumption are well known and need to be assessed on a personalized basis, as is the case for opiate use. ${ }^{52}$ Moreover, the careful review of substance use is required, as multiple substances that are misused are highly associated with impaired cognitive functions (methamphetamines, ketamine). ${ }^{53,54}$

The high and rising rates of cannabis use across jurisdictions, fueled in part by changing legal, cultural, social, and political factors, is resulting in a larger number of individuals reporting cannabis consumption during the past year. ${ }^{55}$ Cannabis (notably Tetrahydrocannabinol (THC)), is established as anticognitive in both healthy and clinical populations, with the extent of reversibility variably reported. ${ }^{56}$ In addition to anticognitive effects, phytocannabinoids like THC are also associated with amotivation and incident mental disorders (e.g., schizophrenia), which are also associated with cognitive impairment. ${ }^{57}$ Circumstances in which cognitive deficits persist, despite ruling out and/or managing any of the foregoing secondary causes, invite the need for additional screening for cognitive function as required. A further issue for consideration is the reporting of null findings in studies of approved antidepressant medication and its effects on cognition. As has been pointed out, clear evidence of a lack of positive effects should be considered in the context of the selected measures' capacity to demonstrate assay sensitivity. $^{58}$

\section{Tools for measuring cognition}

Multiple screening, diagnostic, and measurement tools for cognitive functions are available and/or published in the biomedical literature. Available instruments vary in their administration (e.g., patient-administered), interface (e.g., digital), duration, subjective and/or objective measurements, domains evaluated, proprietary and copyright properties, requirement for expert interpretation, scalability and appropriateness for point-of-care utilization, psychometric properties, validation procedures, and cultural/regional/country sensitivity. No single tool stands out as the gold standard akin to the sphygmomanometer for blood pressure evaluation. Notwithstanding, guiding principles in selecting a screening tool for cognitive dysfunction include that it is patient-administered, brief, digital and interoperable with other digital platforms, available at point-of-care, and free of cost; integrates both subjective and objective cognitive performance; has appropriate psychometric properties; and provides actionable information immediately. ${ }^{59,60}$

A recently validated short and feasible tool for assessment of cognition in psychotic and depressive disorders is the SCIP (Screen for Cognitive Impairment in Psychiatry). ${ }^{61}$ The SCIP is a brief cognitive screening tool consisting of five short objective tests of cognition that can be administered in 10-15 minutes quantifies difficulties with verbal working memory, verbal learning and memory, verbal fluency, and psychomotor speed and has high decision validity in patients with mood disorders (i.e., high sensitivity and specificity for cognitive impairment).

Another tool is the THINC-it tool. The THINC-it tool is free of charge, digitalized, patient administered, and 
has been validated as a screening tool in adults with Major Depressive Disorder (https://thinc.progress.im/). The THINC-it tool can be used at point of care and provides the end user with an easy to translate assessment and can be used as a repeat measure across time, in adults with Major Depressive Disorder. The MMSE and the MOCA are well-known tools for dementia screening, ${ }^{62-64}$ and their use, along with establishment of functional impairment in ADLs and IADLs, can be valuable when a possible major cognitive disorder is suspected. However, the MMSE and MOCA may not be sufficient to identify cognitive dysfunction in older populations with higher cognitive baseline or younger populations with diagnoses that have cognitive impairment as one of many possible symptoms (e.g., bipolar disorder, schizophrenia, diabetes mellitus, hypothyroidism). A point to be emphasized is the importance of an age-informed approach to the screening tool selected for cognitive impairment (i.e., the MMSE/MOCA is suitable for older patients, but not younger patients). ${ }^{65}$

Ongoing research endeavors to identify new technological approaches in assessing cognitive dysfunction may provide more objective markers to supplement clinical assessment. Functional near-infrared spectroscopy (fNIRS) is a noninvasive neuroimaging technology that maps the functions of the cerebral cortex by measuring hemodynamics. ${ }^{66}$ Assessment of hemodynamics by fNIRS during cognitive tasks can be a promising biomarker in personalized psychiatric practice.

If a major cognitive disorder is expected, appropriate and thorough workup is encouraged (e.g., Alzheimer's disease). ${ }^{67}$ If an individual exhibits deficits in two or more cognitive domains with no functional impairment, by definition, the individual has a mild cognitive disorder and should be prospectively evaluated for the possible declaration of major cognitive disorder. Subjective cognitive impairment with no objective findings and no functional impairment indicates that the individual has subjective cognitive complaints/age-related cognitive decline. Reassurance and follow-up are warranted.

Multiple screening/measurement tools for cognitive function have been validated for specific and/or select mental/medical disorders. ${ }^{68}$ When major or mild cognitive disorder is not in the differential diagnosis, and agerelated cognitive decline has been ruled out, it would not be unreasonable for cognitive functions to be measured with a tool with exceptional psychometric properties in healthy controls (age, sex, education-adjusted). It would be impossible to validate any tool across the plethora of medical conditions characterized by cognitive complaints. Indeed, preference is given to those screening tools validated in a specific disorder or disease state. The widespread availability of mobile phones, health-related apps, and digital literacy provides a unique opportunity for screening and surveillance of cognitive function. However, relatively few digital solutions have a rigorous and controlled evidence-based approach supporting their validity as appropriate screening and monitoring tools for cognition in the general and medical population. ${ }^{69}$

When screening provides evidence that cognitive impairment is present and impacting role functioning, steps can be taken to address ways to minimize, reverse, and adapt to the dysfunction. This starts with sharing the results of the screening and enlisting interpersonal and/or professional support needed to start a treatment plan.

\section{Conclusion}

Cognitive dysfunction is a common complaint, a reason for high health-care utilization, a source of patient distress, and a principal determinant of health-care outcomes. It is also established that cognitive dysfunction is a transdiagnostic abnormality with distinct and overlapping phenotypic characteristics across mental/ medical disorders. The foregoing provides the basis for health-care providers to prioritize cognitive dysfunction as a primary therapeutic target. As assessment and measurement have been demonstrated to improve health outcomes across chronic diseases, it is a testable hypothesis that screening and measuring cognitive dysfunction in the health-care ecosystem improves health outcomes among those affected. In the interim, adopting a systematic, coherent, and comprehensive approach to cognitive function evaluation that is pragmatic, patient-centric, comprehensive, and evidencebased is warranted.

\section{Disclosures}

Roger S. McIntyre reports grants from Allergan, AstraZeneca, Bristol-Myers, Janssen-Ortho, Lundbeck, Otsuka, Purdue, Pfizer, Shire, Sunovion, Neurocrine, and Takeda outside the submitted work.

Nicole Anderson, Bernhard T. Baune, Katherine Burdick, Phillipe Fossati, Rodrigo B. Mansur, Alice Medalia, Tanya Ramey, and Joshua D. Rosenblat have nothing to disclose.

Elisa Brietzke reports personal fees from DaiichiSakyo outside the submitted work.

Philip Gorwood reports grants from Eli Lilly, Ethypharm, and Servier and personal fees from Janssen, Lilly, Lundbeck, Otsuka, and Servier outside the submitted work. 
Catherine Harmer reports personal fees from P1vital, Lundbeck, Servier, and Pfizer, grants from UCB, and grants and personal fees from Johnson and Johnson outside the submitted work.

John Harrison reports personal fees from AbbVie, Amgen, Anavex, Astra Zeneca, Avonex, Avraham, Axon Neuroscience, Axovant, Biogen Idec, Boehringer Ingelheim, Bracket, Catenion, CRF Health, DeNDRoN, Eisai, Eli Lilly, Enzymotec, ePharmaSolutions, Forum Pharma, GfHEU, Heptares, Janssen AI, Johnson \& Johnson, Kaasa Health, Kyowa Hakko Kirin, MedAvante, Merck, Mind Agilis, MyCognition, Neurim, Neurocog, personal fees and other from Neurotrack, Novartis, Nutricia, Orion Pharma, Pfizer, Pharmanet/i3, Prana Biotech, PriceSpective, Probiodrug, Prophase, Prostrakan, Regeneron, Reviva, Roche, Sanofi, Servier, Takeda, vTv Therapeutics, Velacor, Lundbeck, Compass Pathways, G4X Discovery, Cognition Therapeutics, and AlzeCure outside the submitted work.

Philip Harvey reports personal fees from Akili, Biogen, Allergan, Boehringer-Ingelheim, Forum Pharma, Genentech, Intracellular Therapies, Lundbeck, Minerva Pharma, Otsuka Digital Health, Sanofi, Sunovion (DSP), and Teva, grants and personal fees from Takeda, grants from Stanley Medical Research Foundation, and other from Neurocog Trials during the conduct of the study.

Kamilla Miskowiak reports personal fees from Lundbeck and Allergan outside the submitted work.

Carola Rong reports personal fees from EOCI Pharmacomm outside the submitted work.

Allan Young reports grants from Janssen and personal fees from Lundbeck, Livanova, and Sunovion outside the submitted work.

Stephen M. Stahl reports grants from Acadia, Alkermes, AssureX, Astra Zeneca, Arbor Pharmaceuticals, Avanir, Axovant, Biogen, Braeburn Pharmaceuticals, BristolMyer Squibb, Celgene, CeNeRx, Cephalon, Dey, Eli Lilly, EnVivo, Forest, Forum, GenOmind, Glaxo Smith Kline, Intra-Cellular Therapies, ISSWSH, Janssen, JayMac, Jazz, Lundbeck, Merck, Mylan, Neurocrine, Neuronetics, Novartis, Otsuka, PamLabs, Pfizer, Reviva, Roche, Sepracor, Servier, Shire, Sprout, Sunovion, TMS NeuroHealth Centers, Takeda, Teva, Tonix, Vanda, Valeant and Wyeth and personal fees from Acadia, Adamas, Alkermes, Allergan, Arbor Pharmaceutcials, AstraZeneca, Avanir, Axovant, Axsome, Biogen, Biomarin, Biopharma, Celgene, Concert, ClearView, DepoMed, Dey, EnVivo, Ferring, Forest, Forum, Genomind. Innovative Science Solutions, Intra-Cellular Therapies, Janssen, Jazz, Lilly, Lundbeck, Merck, Neos, Novartis, Noveida, Orexigen, Otsuka, PamLabs, Perrigo, Pfizer, Pierre Fabre, Reviva, Servier, Shire, Sprout, Sunovion, Taisho, Takeda, Taliaz, Teva, Tonix, Trius, Vanda, and Viforpharma, and other from RCT Logic and Genomind outside the submitted work.

\section{REFERENCES:}

1. McIntyre RS, et al. Cognitive deficits and functional outcomes in major depressive disorder: determinants, substrates, and treatment interventions. Depress Anxiety. 2013; 30(6): 515-527.

2. Millan MJ, et al. Cognitive dysfunction in psychiatric disorders: characteristics, causes and the quest for improved therapy. Nat Rev Drug Discov. 2012; 11(2): 141-168.

3. Murray CJL, Lopez AD. Measuring global health: motivation and evolution of the Global Burden of Disease Study. Lancet. 2017; 390 (10100): 1460-1464.

4. Razzouk D. Mental Health Economics: The Costs and Benefits of Psychiatric Care. Springer, 2017.

5. Insel TR. Assessing the economic costs of serious mental illness. Am J Psychiatry. 2008; 165(6): 663-665.

6. GBD 2016 DALYs and HALE Collaborators. Global, regional, and national disability-adjusted life-years (DALYs) for 333 diseases and injuries and healthy life expectancy (HALE) for 195 countries and territories, 1990-2016: a systematic analysis for the Global Burden of Disease Study 2016. Lancet. 2017; 390(10100): 12601344 .

7. Sokratis $\mathrm{P}$, Ion B, Alexandra E, et al. Cognition and driving behavior in neurodegenerative disorders. Front Hum Neurosci. $2016 ; 10$.

8. Greenberg PE, FournierA-A, Sisitsky T, et al. The economic burden of adults with major depressive disorder in the United States (2005 and 2010). J Clin Psychiatry. 2015; 76(2): 155-162.

9. McIntyre RS, et al. The prevalence and clinical characteristics associated with Diagnostic and Statistical Manual Version-5-defined anxious distress specifier in adults with major depressive disorder: results from the International Mood Disorders Collaborative Project. Ther Adv Chronic Dis. 2016; 7(3): 153-159.

10. Lee $\mathrm{Y}$, et al. Efficacy of antidepressants on measures of workplace functioning in major depressive disorder: a systematic review. J Affect Disord. 2017; 227: 406-415.

11. Baune BT, Malhi GS. A review on the impact of cognitive dysfunction on social, occupational, and general functional outcomes in bipolar disorder. Bipolar Disord. 2015; 17 (suppl 2): 41-55.

12. Baune BT, Renger L. Pharmacological and non-pharmacological interventions to improve cognitive dysfunction and functional ability in clinical depression-a systematic review. Psychiatry Res. 2014; 219(1): 25-50.

13. Vinberg M, Miskowiak KW, Kessing LV. Impairment of executive function and attention predicts onset of affective disorder in healthy high-risk twins. J Clin Psychiatry. 2013; 74(8): e747-53, Aug. 2013.

14. Miskowiak KW, et al. Methodological recommendations for cognition trials in bipolar disorder by the International Society for Bipolar Disorders Targeting Cognition Task Force. Bipolar Disord. 2017; 19(8): 614-626.

15. Papakostas GI, Culpepper L. Understanding and managing cognition in the depressed patient. J Clin Psychiatry. 2015; 76(4): 418-425.

16. McIntyre RS, Lee Y, Mansur RB. Treating to target in major depressive disorder: response to remission to functional recovery. CNS Spectr. 2015; 20(suppl 1): 20-30; quiz 31.

17. Culpepper L. Improving patient outcomes in depression through guideline-concordant, measurement-based care. J Clin Psychiatry. 2013; 74(4): e07.

18. Insel T, et al. Research Domain Criteria (RDoC): toward a new classification framework for research on mental disorders. Am J Psychiatry. 20101; 167(7): 748-751.

19. McTeague LM, Huemer J, Carreon DM, et al. Identification of common neural circuit disruptions in cognitive control across psychiatric disorders. Am J Psychiatry. 2017; 174(7): 676-685 
20. McTeague LM, Goodkind MS, Etkin A. Transdiagnostic impairment of cognitive control in mental illness. J Psychiatr Res. 2016; 83: $37-46$.

21. Lin GR, McCormick JI, Dhe-Paganon S, et al. Role of specific acidic lipids on the reconstitution of $\mathrm{Na}(+)$-dependent amino acid transport in proteoliposomes derived from Ehrlich cell plasma membranes. Biochemistry. 1990; 29(19): 4575-4581.

22. Insel TR. The NIMH Research Domain Criteria (RDoC) Project: precision medicine for psychiatry. Am J Psychiatry. 2014; 171(4): 395-397.

23. McIntyre RS, Johe K, Rong C, Lee Y. The neurogenic compound, NSI-189 phosphate: a novel multi-domain treatment capable of procognitive and antidepressant effects. Expert Opin Investig Drugs. 2017; 26(6): 767-770.

24. McIntyre RS. A vision for drug discovery and development: novel targets and multilateral partnerships. Adv Ther. 2014; 31(3): 245-246.

25. McIntyre RS, Lee Y. Cognition in major depressive disorder: a "Systemically Important Functional Index" (SIFI). Curr Opin Psychiatry. 2016; 29(1): 48-55.

26. Doran CM, Kinchin I. A review of the economic impact of mental illness. Aust Health Rev. 2017. doi: 10.1071/AH16115.

27. Patel V, et al. Global priorities for addressing the burden of mental, neurological, and substance use disorders. In Patel V, Chisholm D, Dua T, et al., eds. Mental, Neurological, and Substance Use Disorders: Disease Control Priorities. Vol. 4. 4th ed. Washington, DC: International Bank for Reconstruction and Development/ World Bank; 2016.

28. Paddick S-M, Gray WK, McGuire J, et al. Cognitive screening tools for identification of dementia in illiterate and low-educated older adults, a systematic review and meta-analysis. Int Psychogeriatr. 2017; 29(6): 897-929.

29. Sheffield JM, et al. Transdiagnostic associations between functiona brain network integrity and cognition. JAMA Psychiatry. 2017; 74 (6): 605-613.

30. McIntyre RS, Cha DS, Soczynska JK. Cognition in Major Depressive Disorder. 2014.

31. McCoy TH, Castro VM, Rosenfield HR, et al. A clinical perspective on the relevance of research domain criteria in electronic health records. Am J Psychiatry. 2015; 172(4): 316-320.

32. Pranckeviciene A, Deltuva VP, Tamasauskas A, Bunevicius A. Association between psychological distress, subjective cognitive complaints and objective neuropsychological functioning in brain tumor patients. Clin Neurol Neurosurg. 2017; 163: 18-23.

33. Cacciamani F, et al. Low cognitive awareness, but not complaint, is a good marker of preclinical Alzheimer's disease. J Alzheimers Dis. 2017; 59(2): 753-762.

34. Srisurapanont M, Suttajit S, Eurviriyanukul K, Varnado P. Discrepancy between objective and subjective cognition in adults with major depressive disorder. Sci Rep. 2017; 7(1); 3901.

35. Golan D, et al. The impact of subjective cognitive fatigue and depression on cognitive function in patients with multiple sclerosis. Mult Scler. 2018; 24(2): 196-204.

36. Mansur RB, et al. Determinants of cognitive function in individuals with type 2 diabetes mellitus: A meta-analysis. Ann Clin Psychiatry. 2018; 30(1): 38-50.

37. Demant KM, Vinberg M, Kessing LV, Miskowiak KW. Assessment of subjective and objective cognitive function in bipolar disorder: correlations, predictors and the relation to psychosocial function. Psychiatry Res. 2015; 229(1-2): 565-571.

38. Kinsinger SW, Lattie E, Mohr DC. Relationship between depression, fatigue, subjective cognitive impairment, and objective neuropsychological functioning in patients with multiple sclerosis. Neuropsychology. 2010; 24(5): 573-580.
39. Ott CV, et al. Screening for cognitive dysfunction in unipolar depression: validation and evaluation of objective and subjective tools. J Affect Disord. 2016; 190: 607-615.

40. Shi C, et al. Reliability and validity of Chinese version of perceived deficits questionnaire for depression in patients with MDD. Psychiatry Res. 2017; 252: 319-324.

41. Ventura J, et al. The Cognitive Assessment Interview (CAI): development and validation of an empirically derived, brief interview-based measure of cognition. Schizophr Res. 2010; 121 (1-3): 24-31.

42. Richards M, Wadsworth MEJ. Long term effects of early adversity on cognitive function. Arch Dis Child. 2004; 89(10): 922-927, 2004

43. Kumar RG, Gao S, Juengst SB, et al. The effects of post-traumatic depression on cognition, pain, fatigue, and headache after moderate-to-severe traumatic brain injury: a thematic review. Brain Inj. 2018; 32(4): 383-394.

44. Roiser JP, Sahakian BJ. Hot and cold cognition in depression. CNS Spectr. 2013; 18(3): 139-149.

45. McIntyre RS, Cha DS, Soczynska JK. "Hot" versus "cold" cognition. In Cognition in Major Depressive Disorder. 2014: 21-30.

46. Fenesy MC, Lee SS. Association of ADHD and executive functioning with childhood depression. J Atten Disord. 2017; 108705471772398 .

47. Ritchie M, Yeap BB. Thyroid hormone: influences on mood and cognition in adults. Maturitas. 2015; 81(2): 266-275.

48. Mansur RB, et al. Determinants of cognitive function in individuals with type 2 diabetes mellitus: a meta-analysis. Ann Clin Psychiatry. 2018; 30(1): 38-50.

49. Lowe CJ, Safati A, Hall PA. The neurocognitive consequences of sleep restriction: a meta-analytic review. Neurosci Biobehav Rev. 2017; 80: 586-604

50. Hindmarch I. Cognition and anxiety: the cognitive effects of antianxiety medication. Acta Psychiatr Scand. 1998; 98(suppl 393): 89-94.

51. Qidwai JC, Watson GS, Weiler JM. Sedation, cognition, and antihistamines. Curr Allergy Asthma Rep. 2002; 2(3): 216-222.

52. Ieong HF-H, Yuan Z. Resting-state neuroimaging and neuropsychological findings in opioid use disorder during abstinence: a review. Front Hum Neurosci. 2017; 11:169.

53. Potvin S, Pelletier J, Grot S, et al. Cognitive deficits in individuals with methamphetamine use disorder: a meta-analysis. Addict Behav. 2018; 80: $154-160$

54. Giorgetti R, Marcotulli D, Tagliabracci A, Schifano F. Effects of ketamine on psychomotor, sensory and cognitive functions relevant for driving ability. Forensic Sci Int. 2015; 252: 127-142.

55. Carliner H, Brown QL, Sarvet AL, Hasin DS. Cannabis use, attitudes, and legal status in the U.S.: a review. Prev Med. 2017; 104: 13-23.

56. Volkow ND, et al. Effects of cannabis use on human behavior, including cognition, motivation, and psychosis: a review. JAMA Psychiatry. 2016; 73(3): 292-297.

57. Rong $\mathrm{C}$, et al. Cannabidiol in medical marijuana: research vistas and potential opportunities. Pharmacol Res. 2017; 121: 213-218.

58. Harrison JE, Lam RW, Baune BT, McIntyre RS. Selection of cognitive tests for trials of therapeutic agents. Lancet Psychiatry. 2016; 3(6); 499.

59. Baune BT, et al. Cognition in depression: can we THINC-it better? J Affect Disord. 2018; 225: 559-562.

60. Ragguett R-M, Cha DS, Kakar R, et al. Assessing and measuring cognitive function in major depressive disorder. Evid Based Ment Health. 2016; 19(4): 106-109.

61. Miskowiak KW, et al. Assessing and addressing cognitive impairment in bipolar disorder: the International Society for Bipolar Disorders Targeting Cognition Task Force recommendations for clinicians. Bipolar Disord. 2018; 20(3): 184-194. 
62. Freitas S, Santana I, Simoes MR. The sensitivity of the MoCA and MMSE to cognitive decline: a longitudinal study. Alzheimers Dement. 2010; 6(4): S353-S354.

63. Julayanont P, Nasreddine ZS. Montreal Cognitive Assessment (MoCA): concept and clinical review. In Larner AJ, ed. Cognitive Screening Instruments. London: Springer; 2016: 139-195.

64. Finney GR, Minagar A, Heilman KM. Assessment of mental Status Neurol Clin. 2016; 34(1): 1-16.

65. Baune BT, Miller R, McAfoose J, et al. The role of cognitive impairment in general functioning in major depression. Psychiatry Res. 2010; 176(2-3): 183-189.
66. Lai CYY, Ho CSH, Lim CR, Ho RCM. Functional near-infrared spectroscopy in psychiatry. BJPsych Advances. 2017; 23(5): 324-330.

67. Rogan S, Lippa CF. Alzheimer's disease and other dementias: a review. Am J Alzheimers Dis Other Demen. 2002; 17(1): 11-17.

68. Pan $\mathrm{Z}$, et al. Pharmacological treatment of cognitive symptoms in major depressive disorder. CNS Neurol Disord Drug Targets. 2017; 16(8): 891-899.

69. Chan S, Godwin H, Gonzalez A, et al. Review of use and integration of mobile apps into psychiatric treatments. Curr Psychiatry Rep. 2017; 19(12); 96. 